\title{
Solução de problemas de Helmholtz pelo Método dos Elementos de Contorno com solução fundamental dependente da frequência.
}

\section{Carlos Friedrich Loeffler}

Programa de Pós-Graduação em Engenharia Mecânica, UFES, Vitoria, ES

\section{Pedro Vinicius Moreira Pereira}

Programa de Pós-Graduação em Engenharia Mecânica, UFES, Vitoria, ES

\section{Luciano de Oliveira Castro Lara ${ }^{3}$}

Programa de Pós-Graduação em Engenharia Mecânica, UFES, Vitoria, ES

\begin{abstract}
Resumo. Com a meta de validar uma técnica de solução para problemas de Helmholtz, o Método dos Elementos de Contorno com Integração Direta (MECID), que emprega funções de base radial para aproximar o termo de inércia, é utilizado para resolver numericamente problemas regidos pela equação de Helmholtz. Para comparar os resultados, utiliza-se a formulação tradicional do Método dos Elementos de Contorno que emprega a solução fundamental do problema correlato, que tem como um dos argumentos da solução fundamental a frequência de excitação. Os exemplos resolvidos com ambas as formulações do Método dos Elementos de Contorno serão comparados com soluções analíticas.
\end{abstract}

Palavras-chave. Método dos Elementos de Contorno, Equação de Helmholtz, Solução fundamental dependente da frequência.

\section{Introdução}

O Método dos Elementos de Contorno (MEC) tem excelente desempenho nas aplicações onde o campo de variáveis é escalar e estacionário, nos quais os operadores que caracterizam matematicamente a equação de governo são auto-adjuntos [1]. Muitas formulações do MEC são dependentes da solução fundamental [2] associada ao problema, que para alguns casos se verifica ser difícil de obter. Entretanto, para a equação de Helmholtz, esta é conhecida e dependente da frequência de excitação.

Dada à equação de Helmholtz:

\footnotetext{
1 carlosloeffler@bol.com.br

2 pedrovinicius012@gmail.com

3castrolara@hotmail.com
} 


$$
\nabla^{2} u+k^{2} u=0
$$

segundo o procedimento padrão do MEC, obtem-se a forma integral inversa:

$$
\int_{\Omega} u u_{, i i}^{*} d \Omega+k^{2} \int_{\Omega} u u^{*} d \Omega=\int_{\Omega} u\left[u_{, i i}^{*}+k^{2} u^{*}\right] d \Omega=c(\xi) u(\xi),
$$

onde $\mathrm{k}$ é a frequência e para contorno suave $c(\xi)=\alpha / 2 \pi$ com $\alpha$ sendo o ângulo formado pelo contorno no ponto $\xi$, a solução fundamental $u^{*}$ deve atender:

$$
\left(u_{, i i}^{*}+k^{2}\right)=-\Delta(\xi ; x)
$$

Desta forma, a solução fundamental é:

$$
u^{*}(\xi ; x)=\frac{1}{2 \pi \mu} K_{0}
$$

onde $\mathrm{K}_{0}$ é a função de Bessel modificada da segunda espécie e ordem zero.

Discretizando a equação integral através de elementos de contorno lineares e resolvendo-a para diferentes valores de $\mathrm{k}$, consegue-se estabelecer um espectro de resposta no qual os valores das frequências naturais podem ser identificados e comparados com os valores dados por expressões analíticas e também por diferentes métodos numéricos. Desta forma serão realizadas às comparações entre o procedimento denominado de Método dos Elementos de contorno com Integração Direta (MECID), que já foi implementada com êxito nos problemas de Poisson [3, 4], a solução analítica e o MEC com a solução fundamental correlata ao problema de Helmholtz.

\section{Referências}

[1] C. A. Brebbia, J. C. Telles and L. C. Wrobel, Boundary Element Techniques, Springer-Verlag, (1984).

[2] C. A. Brebbia and S. Walker, Boundary Element Techniques in Engineering. London: Newnes-Butterworths, Cap. II, (1980).

[3] A. L. Cruz, Modelagem Direta de Integrais de Domínio usando Funções de Base Radial no contexto do Método dos Elementos de Contorno, Dissertação de Mestrado em Engenharia Mecânica UFES, (2012).

[4] L. Zamprogno, Utilização de funções Radiais de Base Compacta na Modelagem Direta de Integrais de Domínio com o Método dos Elementos de Contorno, Dissertação de Mestrado em Engenharia Mecânica UFES, (2013). 